\title{
Revised Equations to Estimate Glomerular Filtration Rate from Serum Creatinine and Cystatin C in China
}

\author{
Min Yang ${ }^{a}$ Yonghua Zou ${ }^{b}$ Tong $L^{c}{ }^{c}$ Yule Nan ${ }^{c}$ Jianying $\mathrm{Niu}^{a}$ \\ Xin $\mathrm{Du}^{\mathrm{d}}$ Yong Gu${ }^{\mathrm{a}}$ e \\ aDivision of Nephrology, Shanghai Fifth People's Hospital, Fudan University, Shanghai, PR \\ China; ${ }^{b}$ Outpatient Department, Nanjing Medical University, Nanjing, PR China; 'Division of \\ Nuclear Medicine, Shanghai Fifth People's Hospital, Fudan University, Shanghai, PR China; \\ dDivision of Nephrology, Nanjing First Hospital, Nanjing Medical University, Nanjing, \\ PR China; e Division of Nephrology, Huashan Hospital, Fudan University, Shanghai, PR China
}

\section{Keywords}

Serum cystatin C . Serum creatinine - Estimated glomerular filtration rate $\cdot$ Chronic kidney disease

\begin{abstract}
Aim: Our previous study demonstrated that the cystatin C-based chronic kidney disease (CKD)-EPI equation and combined by serum creatinine (CKD-EPIscr-cys) had better capability to accurately evaluate glomerular filtration rate in the CKD participants. Considering that the accuracy of estimated glomerular filtration rate (eGFR) remains less ideally, it is essential to modify the equation by including the Chinese eGFR racial factor in order to improve its performance. Methods: Two prospective cohorts were enrolled in 2 medical centers. New equations were developed in 529 participants and validated in 313 participants. Reference glo-

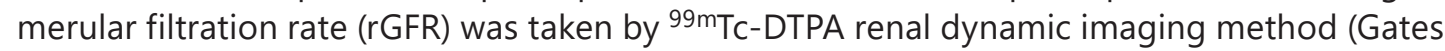
method). The primary outcomes of this study were bias, precision (interquartile range of difference [IQR]), and accuracy (the proportion of eGFR within 30\% of rGFR [P30] and root mean square error [RMSE]) of eGFR versus rGFR. Results: In a development data set, Chinese coefficients for CKD-EPIscr (C-CKD-EPIsCr), CKD-EPIcys (C-CKD-EPIcys), and CKD-EPIscr-cys (CCKD-EPIscr-cys) were $0.871,0.879$, and 0.891 , respectively. In a validation data set, C-CKDEPIcys was the most accurate with highest P30 value (62.3\%), relative lowest IQR (15.45), and RMSE (0.80) among 6 equations, though the bias of C-CKD-EPIcys was not better than CKD-
\end{abstract}




\section{Kidney \\ Blood Pressure \\ Research}

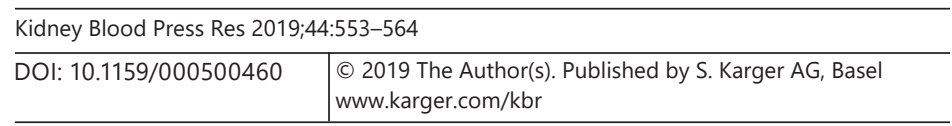

Yang et al.: Revised Equations to Estimate GFR from Scr and Cystatin C in China

EPIcys. C-CKD-EPIscr and C-CKD-EPIscr-cys equations were improved in bias $(p<0.001)$, precision, and accuracy ( $p=0.004$ and $<0.001$ for P30) compared with CKD-EPIscr and CKDEPIscr-cys. Conclusion: C-CKD-EPIcys was the most accurate with the highest P30 value, relative lowest IQR, and RMSE among 6 equations. C-CKD-EPIsCr and C-CKD-EPIscr-cys equations were improved in bias, precision, and accuracy. Other external validation of these equations is needed.

(C) 2019 The Author(s)

Published by S. Karger AG, Basel

\section{Introduction}

Chronic kidney disease (CKD) is a global public health problem [1]. The prevalence of CKD in adults in China was 10.8\% [2]. Awareness of CKD is only $10.04 \%$ from a national crosssectional survey in China [3]. CKD is characterized by decreased estimated glomerular filtration rate (eGFR) and/or increased albuminuria, present for $>3$ months, and is associated with adverse outcomes (all-cause mortality, acute kidney injury, and end-stage renal disease), independent of hypertension and diabetes [4], age [5], or sex [6]. CKD may carry a coronary heart disease risk similar to that of diabetes. The estimated lifetime risk of CKD stage 3a was $>50 \%$ [7], higher than those for diabetes (33-39\%), coronary heart disease (32-49\%), and invasive cancer (38-45\%) [8].

eGFR is a primary means of screening for CKD in epidemiological investigations. Our previous study [9] demonstrated that the CKD-EPI equation combined by serum creatinine and cystatin C (CKD-EPIscr-cys) [10] had better capability to accurately evaluate GFR in Chinese ethnic participants with CKD stages 1-2. The application of the cystatin C-based equations may be the optimal one for patients of moderately to severely injured GFR. Considering that the accuracy in the entire range of participants of our study remains less ideally, we suggest that the addition of the Chinese racial factor may improve eGFR performance [9]. In this study, we aimed to add the Chinese racial factor for the improvement of eGFR performance. We compared their bias, precision, and accuracy with the 2012 CKD-EPI equations [10].

\section{Materials and Methods}

\section{Study Design}

A total of 842 Chinese CKD patients were included from 2 general hospitals between August 1, 2009, and December 31, 2017, in 2 megalopolises of China. The criteria of inclusion and exclusion were seen in our previous work [9]. The study was approved by the Institutional Review Boards of Nanjing First Hospital affiliated to Nanjing Medical University (IRB No. 86-025-52271039-21). The ClinicalTrials.gov number was NCT02841371.

\section{Primary Outcomes}

The primary outcomes of this study were bias, precision, and accuracy of eGFR versus reference glomerular filtration rate (rGFR). Bias was defined as the median results of differences between eGFR and rGFR. Precision was defined as the interquartile range (IQR) of the differences between eGFR and rGFR. Accuracy was calculated as the proportion of eGFR within $30 \%$ of rGFR and root mean square error (RMSE) of mean difference between eGFR and rGFR. 
Table 1. Characteristics of the study population

\begin{tabular}{lccc}
\hline & All subjects & Development data set & Validation data set \\
\hline $\begin{array}{l}\text { Number of patients } \\
\text { Age, years }\end{array}$ & 842 & $529(62.8)$ & $313(37.2)$ \\
Gender & $65(29-86)$ & $64(32-85.8)$ & $66(24.6-89)$ \\
$\quad$ Male & $476(56.5)$ & $296(56.0)$ & $180(57.5)$ \\
$\quad$ Female & $366(43.5)$ & $233(44.0)$ & $133(42.5)$ \\
Scr, $\mu \mathrm{mol} / \mathrm{L}$ & $114(43-658)$ & $104(45.2-658)$ & $126.9(36.9-607.2)$ \\
Scys, $\mathrm{mg} / \mathrm{L}$ & $1.45(0.53-4.81)$ & $1.27(0.51-4.74)$ & $1.66(0.71-4.82)$ \\
rGFR, $\mathrm{mL} / \mathrm{min} / 1.73 \mathrm{~m}^{2}$ & $47(9.9-107.5)$ & $54.6(11.4-108.7)$ & $38.8(9.4-95.4)$ \\
\hline
\end{tabular}

Values for continuous variables expressed as median (95\% CI); values for categorical values expressed as number (percentage). Conversion factors for units: serum creatinine in $\mathrm{mg} / \mathrm{dL}$ to $\mathrm{mmol} / \mathrm{L}, \times 88.4$.

Scr, serum creatinine; Scys, serum cystatin C; rGFR, reference glomerular filtration rate (using the ${ }^{99 \mathrm{~m}} \mathrm{Tc}$ DTPA renal dynamic imaging method); eGFR, estimated glomerular filtration rate.

\section{Measurements of Creatinine, Cystatin C, and GFR}

Enzymatic method was used to measure serum creatinine (Scr) concentration, traceable to National Institute of Standards and Technology creatinine standard reference material (SRM 967). Automated particle-enhanced immunonephelometry assay was applied to examine serum cystatin $C$, which was calibrated against the international certified reference material ERM-DA471. All fasting serum samples were assayed on an Olympus AU5400 autoanalyser (Olympus Corporation, Mishima, Japan), in conformity with manufacturer's instructions.

A ${ }^{99 m}$ Tc-DTPA renal dynamic imaging measurement by modified Gates method was taken as the rGFR, calibrated with dual plasma sample ${ }^{99 \mathrm{~m}} \mathrm{Tc}-\mathrm{DTPA}$ GFR, which had been described in previous reports $[9,11,12]$.

\section{Development and Validation of Equations}

The new Chinese coefficient to modify the 2009 and 2012 CKD-EPI equations for Chinese was calculated from the development data set of 529 participants from Shanghai Fifth People's Hospital affiliated to Fudan University. The revised equations were derived in the development data set by using a linear regression model. The revised CKD-EPI equations for Chinese were validated in the validation data set of 313 participants from Nanjing First Hospital affiliated to Nanjing Medical University.

\section{Statistical Analysis}

In 2002, the Kidney Disease Outcomes Quality Initiative guidelines pointed out that at least 100 samples were needed to test the new GFR equation. According to our previous studies [11], with a deviation of 5 for the paired difference (our registered information, ClinicalTrials.gov registration number: NCT02841371), the deviation of the standard deviation 20, power 0.8, alpha $=0.05,2$-sided test, using Wilcoxon paired rank test, with PASS11 software system, the sample size is required in 128 cases. A total of 313 participants were included in the validation data set of this study, so the sample size has met the statistical requirements.

The data sets were not normally distributed ( $p<0.001$, Kolmogorov-Smirnov test); so, nonparametric statistics (Wilcoxon matched-pairs signed-rank test) were carried out. Bias, precision, and accuracy were applied to assess the performance of each equation. Bias was 
Table 2. Equations to predict GFR

\begin{tabular}{|c|c|c|c|c|}
\hline Name & Gender & $\mathrm{Scr}$ & Scys & Equation \\
\hline \multirow[t]{4}{*}{ CKD-EPIscr } & \multirow[t]{2}{*}{ Female } & $\leq 0.7$ & & $144 \times(\mathrm{Scr} / 0.7)^{-0.329} \times 0.993^{\mathrm{age}}$ \\
\hline & & $>0.7$ & & $144 \times(\mathrm{Scr} / 0.7)^{-1.209} \times 0.993^{\text {age }}$ \\
\hline & \multirow[t]{2}{*}{ Male } & $\leq 0.9$ & & $141 \times(\mathrm{Scr} / 0.9)^{-0.411} \times 0.993^{\mathrm{age}}$ \\
\hline & & $>0.9$ & & $141 \times(\mathrm{Scr} / 0.9)^{-1.209} \times 0.993^{\mathrm{age}}$ \\
\hline \multirow[t]{4}{*}{ CKD-EPIcys } & \multirow[t]{2}{*}{ Female } & & $\leq 0.8$ & $133 \times(\text { Scys } / 0.8)^{-0.499} \times 0.996^{\text {age }} \times 0.932$ \\
\hline & & & $>0.8$ & $133 \times(\text { Scys } / 0.8)^{-1.328} \times 0.996^{\text {age }} \times 0.932$ \\
\hline & \multirow[t]{2}{*}{ Male } & & $\leq 0.8$ & $133 \times(\text { Scys } / 0.8)^{-0.499} \times 0.996^{\text {age }}$ \\
\hline & & & $>0.8$ & $133 \times(\text { Scys } / 0.8)^{-1.328} \times 0.996^{\text {age }}$ \\
\hline \multirow[t]{8}{*}{ CKD-EPIscr-cys } & \multirow[t]{4}{*}{ Female } & $\leq 0.7$ & $\leq 0.8$ & $130 \times(\text { Scr } / 0.7)^{-0.248} \times(\text { Scys } / 0.8)^{-0.375} \times 0.995^{\text {age }}$ \\
\hline & & & $>0.8$ & $130 \times(\mathrm{Scr} / 0.7)^{-0.248} \times(\text { Scys } / 0.8)^{-0.711} \times 0.995^{\text {age }}$ \\
\hline & & $>0.7$ & $\leq 0.8$ & $130 \times(\mathrm{Scr} / 0.7)^{-0.601} \times(\mathrm{Scys} / 0.8)^{-0.375} \times 0.995^{\text {age }}$ \\
\hline & & & $>0.8$ & $130 \times(\mathrm{Scr} / 0.7)^{-0.601} \times(\mathrm{Scys} / 0.8)^{-0.711} \times 0.995^{\text {age }}$ \\
\hline & \multirow[t]{4}{*}{ Male } & $\leq 0.9$ & $\leq 0.8$ & $135 \times(\mathrm{Scr} / 0.9)^{-0.207} \times(\text { Scys } / 0.8)^{-0.375} \times 0.995^{\text {age }}$ \\
\hline & & & $>0.8$ & $135 \times(\mathrm{Scr} / 0.9)^{-0.207} \times(\text { Scys } / 0.8)^{-0.711} \times 0.995^{\text {age }}$ \\
\hline & & $>0.9$ & $\leq 0.8$ & $135 \times(\mathrm{Scr} / 0.9)^{-0.601} \times(\mathrm{Scys} / 0.8)^{-0.375} \times 0.995^{\text {age }}$ \\
\hline & & & $>0.8$ & $135 \times(\mathrm{Scr} / 0.9)^{-0.601} \times(\mathrm{Scys} / 0.8)^{-0.711} \times 0.995^{\text {age }}$ \\
\hline \multirow[t]{4}{*}{ C-CKD-EPIscr } & \multirow[t]{2}{*}{ Female } & $\leq 0.7$ & & $125 \times(\text { Scr } / 0.7)^{-0.329} \times 0.993^{\text {age }}$ \\
\hline & & $>0.7$ & & $125 \times(\mathrm{Scr} / 0.7)^{-1.209} \times 0.993^{\mathrm{age}}$ \\
\hline & \multirow[t]{2}{*}{ Male } & $\leq 0.9$ & & $123 \times(\mathrm{Scr} / 0.9)^{-0.411} \times 0.993^{\mathrm{age}}$ \\
\hline & & $>0.9$ & & $123 \times(\mathrm{Scr} / 0.9)^{-1.209} \times 0.993^{\mathrm{age}}$ \\
\hline \multirow[t]{2}{*}{ C-CKD-EPIcys } & & & $\leq 0.8$ & $117 \times(\text { Scys } / 0.8)^{-0.499} \times 0.996^{\text {age }}$ \\
\hline & & & $>0.8$ & $117 \times(\text { Scys } / 0.8)^{-1.328} \times 0.996^{\text {age }}$ \\
\hline \multirow[t]{8}{*}{ C-CKD-EPIscr-cys } & \multirow[t]{4}{*}{ Female } & $\leq 0.7$ & $\leq 0.8$ & $116 \times(\mathrm{Scr} / 0.7)^{-0.248} \times(\text { Scys } / 0.8)^{-0.375} \times 0.995^{\text {age }}$ \\
\hline & & & $>0.8$ & $116 \times(\mathrm{Scr} / 0.7)^{-0.248} \times(\mathrm{Scys} / 0.8)^{-0.711} \times 0.995^{\text {age }}$ \\
\hline & & $>0.7$ & $\leq 0.8$ & $116 \times(\mathrm{Scr} / 0.7)^{-0.601} \times(\mathrm{Scys} / 0.8)^{-0.375} \times 0.995^{\text {age }}$ \\
\hline & & & $>0.8$ & $116 \times(\mathrm{Scr} / 0.7)^{-0.601} \times(\text { Scys } / 0.8)^{-0.711} \times 0.995^{\text {age }}$ \\
\hline & \multirow[t]{4}{*}{ Male } & $\leq 0.9$ & $\leq 0.8$ & $120 \times(\text { Scr } / 0.9)^{-0.207} \times(\text { Scys } / 0.8)^{-0.375} \times 0.995^{\text {age }}$ \\
\hline & & & $>0.8$ & $120 \times(\mathrm{Scr} / 0.9)^{-0.207} \times(\mathrm{Scys} / 0.8)^{-0.711} \times 0.995^{\text {age }}$ \\
\hline & & $>0.9$ & $\leq 0.8$ & $120 \times(\text { Scr } / 0.9)^{-0.601} \times(\text { Scys } / 0.8)^{-0.375} \times 0.995^{\text {age }}$ \\
\hline & & & $>0.8$ & $120 \times(\mathrm{Scr} / 0.9)^{-0.601} \times(\mathrm{Scys} / 0.8)^{-0.711} \times 0.995^{\mathrm{age}}$ \\
\hline
\end{tabular}

Scr was shown as mg/dL; Scys was shown as mg/L; age was shown as years. Scr, serum creatinine; Scys, serum cystatin C; CKD-EPIscr, serum creatinine-based CKD-EPI equation which was developed in 2009; CKD-EPIcys, cystatin C-based CKD-EPI equation which was newly developed in 2012; CKD-EPIscr-cys, serum creatinine and cystatin C-based CKD-EPI equation which was newly developed in 2012; C-CKD-EPIscr, CKD-EPIscr equation modified for China; C-CKD-EPIcys, CKD-EPIcys equation modified for China; C-CKDEPIscr-cys, CKD-EPIscr-cys equation modified for China.

calculated as the median results of differences between eGFR and rGFR. Precision was defined as the IQR of the differences between eGFR and rGFR. Accuracy was defined as the proportion of eGFR within $30 \%$ of rGFR (P30) and RMSE of mean difference between eGFR and rGFR. McNemar test was used to compare the P30 of the C-CKD-EPIscr against the P30 of CKDEPIscr equation and the C-CKD-EPIscr-cys against the P30 of CKD-EPIscr-cys equation. Classical Bland-Altman plot analysis was performed to compare rGFR with eGFR. Curve fitting was implemented by comparison between eGFR and rGFR for each patient graphically by plotting eGFR and the difference (eGFR - rGFR) against rGFR. Data were considered statistically significant at $p<0.05$. SPSS software version 18.0 (SPSS, Chicago, IL, USA) was used to perform all statistical analyses. 
Table 3. Performance of 6 estimating equations compared with rGFR

\begin{tabular}{lllll}
\hline & Bias (median difference, 95\% CI) & $\begin{array}{l}\text { Precision (IQR of } \\
\text { the difference) }\end{array}$ & $\begin{array}{c}\text { Accuracy P30 } \\
\text { Accuracy } \\
\text { (RMSE) }\end{array}$ \\
\hline CKD-EPIscr & $13.20(-21.87$ to 74.45$)(p<0.001)$ & 59.85 & 37.1 & 1.47 \\
CKD-EPIcys & $-1.87(-24.28$ to 36.61) $(p=0.140)$ & 36.32 & 60.6 & 0.87 \\
CKD-EPIscr-cys & $2.86(-21.57$ to 47.63$)(p<0.001)$ & 45.43 & 47.6 & 1.02 \\
C-CKD-EPIscr & $5.03\left(-27.01\right.$ to 55.39) $(p<0.001)^{*}$ & 32.22 & $62.3(p=0.004)^{\#}$ & 1.25 \\
C-CKD-EPIcys & $-2.97(-25.11$ to 31.59) $(p<0.001)$ & 15.45 & $55.3(p<0.001)^{\#}$ & 0.80 \\
C-CKD-EPIscr-cys & $-1.00(-26.27$ to 36.77$)(p=0.475)^{*}$ & 21.12 & 0.89 \\
\hline
\end{tabular}

* The bias of the C-CKD-EPIscr compared with CKD-EPIscr equation, and C-CKD-EPIscr-cys compared with CKD-EPIscr-cys equation $(p<0.001)$.

\# McNemar test was used to compare the P30 of the C-CKD-EPIscr against the P30 of CKD-EPIscr equation and the C-CKDEPIscr-cys against the P30 of CKD-EPIscr-cys equation.

Bias, median difference between eGFR and rGFR; P30, the proportion of eGFR within 30\% of rGFR; RMSE, root mean square error (of mean difference between eGFR and rGFR); CI, confidence interval; IQR, the interquartile range of difference; CKD-EPIscr, serum creatinine-based CKD-EPI equation which was developed in 2009; CKD-EPIcys, cystatin C-based CKD-EPI equation which was newly developed in 2012; CKD-EPIscr-cys, serum creatinine and cystatin C-based CKD-EPI equation which was newly developed in 2012; C-CKD-EPIscr, CKD-EPIscr equation modified for China; C-CKD-EPIcys, CKD-EPIcys equation modified for China; C-CKD-EPIscr-cys, CKD-EPIscr-cys equation modified for China. Wilcoxon matched-pairs signed rank test was used to compare the difference between the eGFR and rGFR ( $p$ value).

\section{Results}

\section{Characteristics of the Study Population}

A total of 842 participants (476 male and 366 female) with CKD were enrolled in this study. Median age, rGFR, Scr, and cystatin C were $65,47 \mathrm{~mL} / \mathrm{min} / 1.73 \mathrm{~m}^{2}, 114 \mu \mathrm{mol} / \mathrm{L}$, and $1.45 \mathrm{mg} / \mathrm{L}$, respectively. The detailed demographic and clinical characteristics of participants were shown in Table 1.

\section{eGFR Equations}

All equations were listed in Table 2. The coefficients to modify CKD-EPIscr, CKD-EPIcys, and CKD-EPIscr-cys for a Chinese population were 0.871 (0.848-0.891 for 95\% CI), 0.879 (0.857-0.897), and 0.891 (0.870-0.909), respectively. The coefficient to modify CKD-EPIcys for Chinese female was 0.999 (0.997-1.000), almost equal to 1.0. For this reason, we omitted the female coefficient of C-CKD-EPIcys. The coefficient to modify CKD-EPIscr-cys for Chinese female was 0.959 (0.936-0.981).

\section{Comparison of Performance of the Equations}

Performance of the equations in the validation data set was summarized in Table 3 , and bias plots of the 6 equations against rGFR were shown in Figure 1. CKD-EPIscr, CKD-EPIscrcys, and C-CKD-EPIscr equations tended to overestimate GFR in the CKD patients, and CKDEPIcys and C-CKD-EPIcys equations tended to underestimate GFR. C-CKD-EPIcys appeared to be the most accurate with highest P30 value (62.3\%), relative lowest IQR (15.45), and RMSE (0.80) among 6 equations, though the bias of C-CKD-EPIcys was not better than CKD-EPIcys. C-CKD-EPIscr and C-CKD-EPIscr-cys equations were improved in bias $(p<0.001)$, precision, and accuracy (P30 and RMSE; $p=0.004$ and $<0.001$ for P30). Bland-Altman analysis demonstrated a consistent result (Fig. 1). 


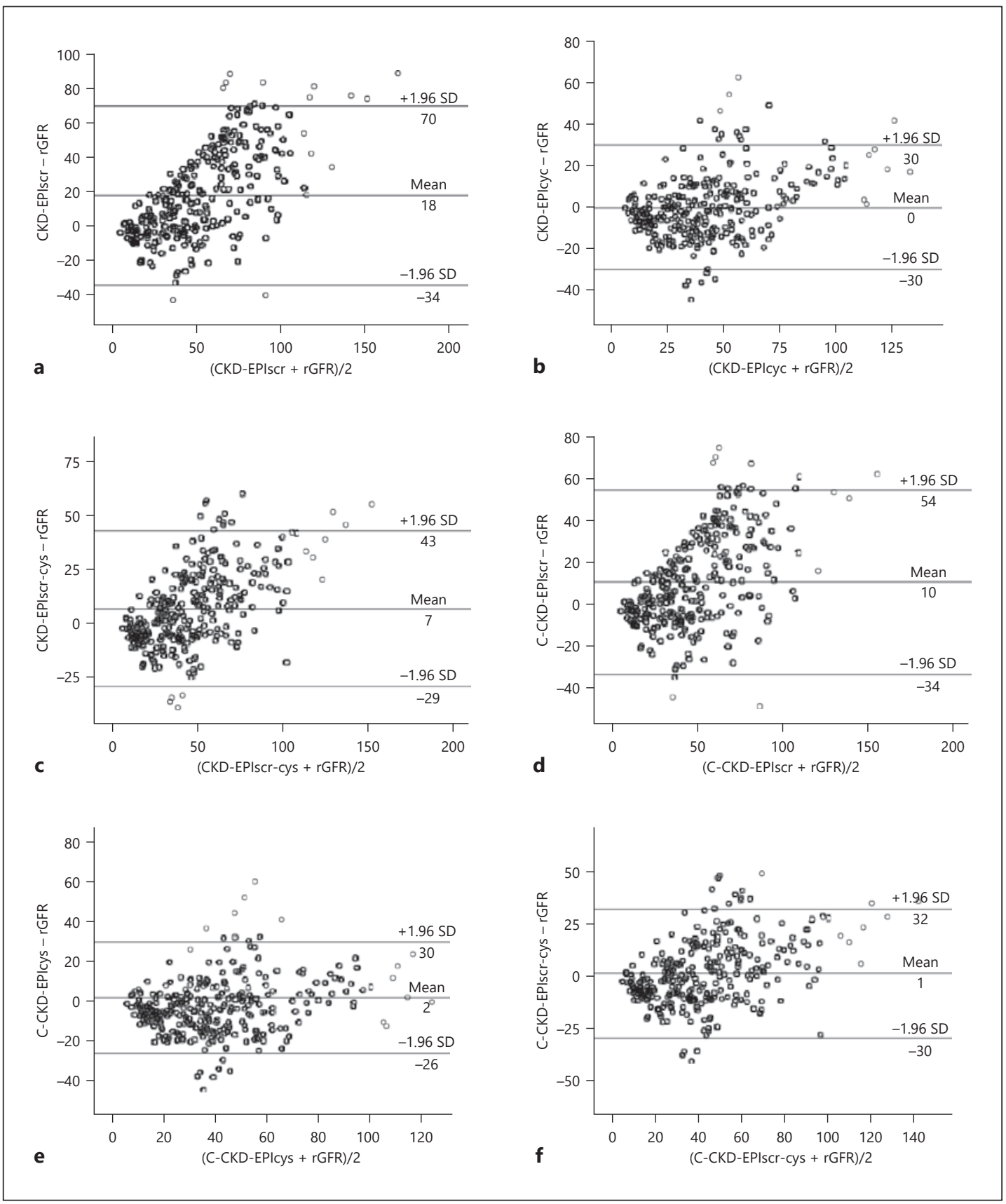

Fig. 1. Bias plots intuitively compare estimated glomerular filtration rate (eGFR) with reference glomerular filtration rate (rGFR). The difference between eGFR and rGFR was regressed against the mean of rGFR and eGFR. The eGFRs were calculated separately from 6 estimating equations. a CKD-EPIscr, serum creatininebased CKD-EPI equation which was developed in 2009; b CKD-EPIcys, cystatin C-based CKD-EPI equation which was newly developed in 2012; c CKD-EPIscr-cys, serum creatinine, and cystatin C-based CKD-EPI equation which was newly developed in 2012; d C-CKD-EPIscr, CKD-EPIscr equation modified for China; e C-CKD-EPIcys, CKD-EPIcys equation modified for China; $\mathbf{f}$ C-CKD-EPIscr-cys, CKD-EPIscr-cys equation modified for China. GFR was measured in $\mathrm{mL} / \mathrm{min} / 1.73 \mathrm{~m}^{2}$. Horizontal solid lines represent the mean difference and \pm 1.96 SD. 


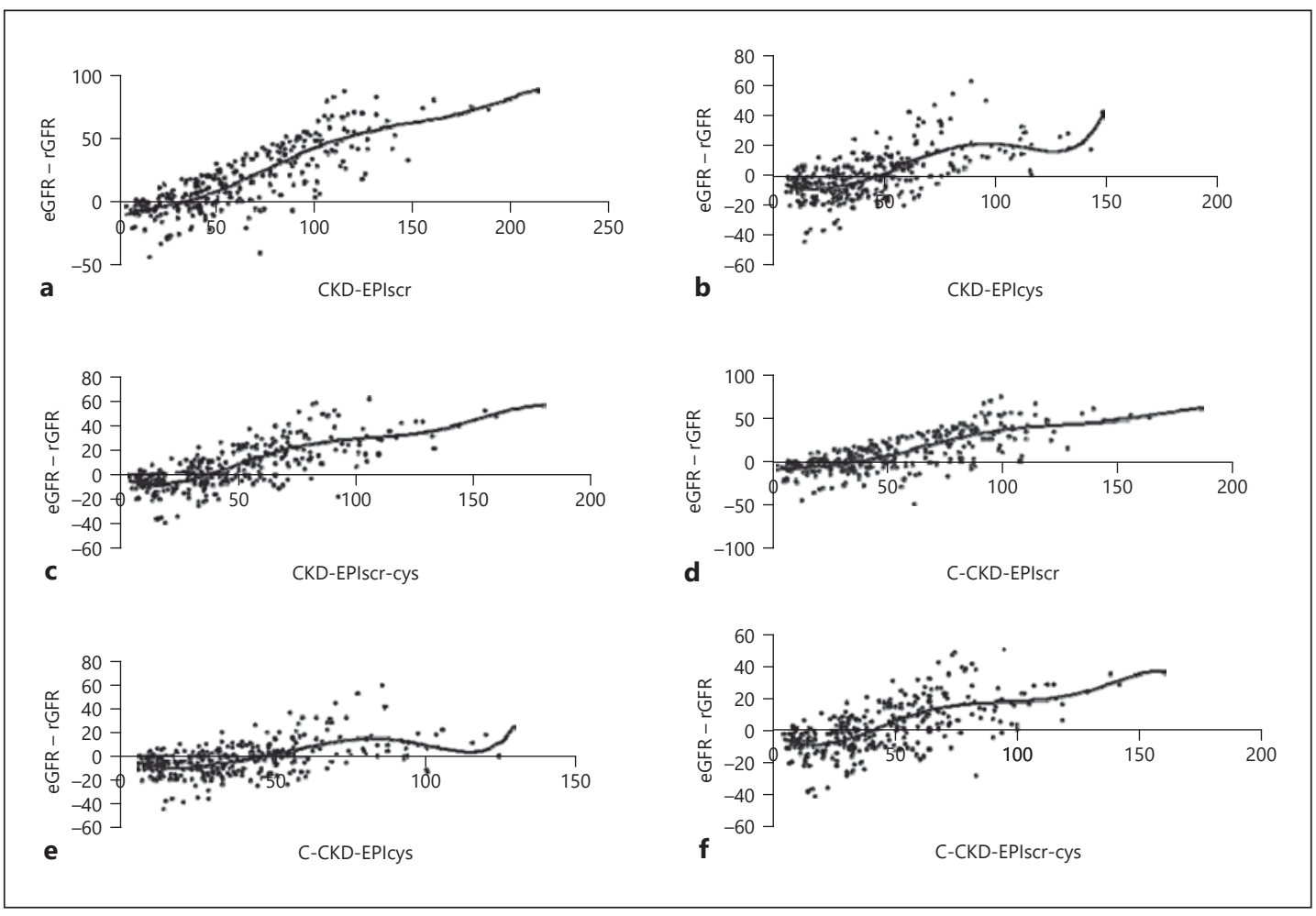

Fig. 2. Relationship between estimated glomerular filtration rate (GFR) and bias (calculated as eGFR - measured GFR) in the validation data set. Solid horizontal line indicates no difference. Smoothed line shows the fit of the data. a CKD-EPIscr, serum creatinine-based CKD-EPI equation which was developed in 2009; b CKD-EPIcys, cystatin C-based CKD-EPI equation which was newly developed in 2012; c CKD-EPIscr-cys, serum creatinine, and cystatin C-based CKD-EPI equation which was newly developed in 2012; d C-CKDEPIscr, CKD-EPIscr equation modified for China; (e) C-CKD-EPIcys, CKD-EPIcys equation modified for China; f C-CKD-EPIscr-cys, CKD-EPIscr-cys equation modified for China. GFR was measured in $\mathrm{mL} / \mathrm{min} / 1.73 \mathrm{~m}^{2}$.

\section{Correlation Between eGFR and rGFR}

The correlation between eGFR and bias was evaluated in the validation data set as shown for each equation in Figure 2. The smoothed lines of C-CKD-EPIscr, C-CKD-EPIcys, and C-CKD-EPIscr-cys equations were upwardly curving and showed similar slopes with the individuals of eGFR $30-90 \mathrm{~mL} / \mathrm{min} / 1.73 \mathrm{~m}^{2}$. Plots of eGFR and rGFR were shown in Figure 3. As the fit of the data shown (Fig. 2, 3), smoothed lines of C-CKD-EPIscr, C-CKD-EPIcys, and C-CKD-EPIscr-cys equations were nearer to zero than compared with CKD-EPIscr, CKDEPIcys, and CKD-EPIscr-cys equations, which means that all 3 modified equations were on the whole improved.

\section{Discussion}

In this study, we added the Chinese racial factor for the improvement of eGFR performance of the 2009 CKD-EPI equation and two 2012 CKD-EPI equations. The new Chinese coefficient to modify the 2012 CKD-EPI equations for Chinese was calculated from the development data set of 529 participants. The revised CKD-EPI equations for Chinese were validated in the validation data set of 313 participants. We compared their bias, precision, and 


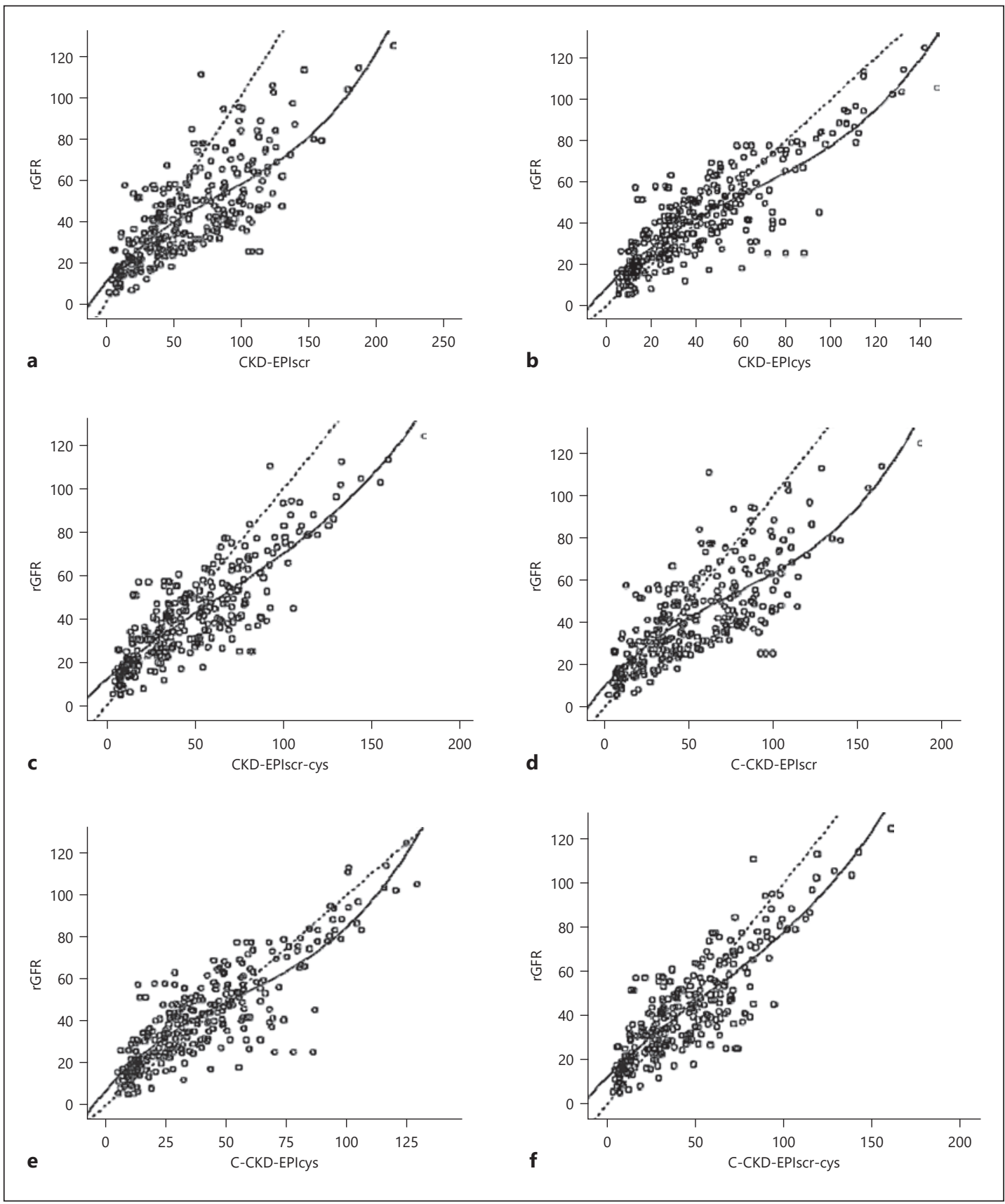

Fig. 3. Correlation between estimated glomerular filtration rate (eGFR) and reference glomerular filtration rate (rGFR) in the validation data set. Smoothed line shows the fit of the data. Dashed line indicates no difference. a CKD-EPIscr, serum creatinine-based CKD-EPI equation which was developed in 2009; b CKDEPIcys, cystatin C-based CKD-EPI equation which was newly developed in 2012; c CKD-EPIscr-cys, serum creatinine, and cystatin C-based CKD-EPI equation which was newly developed in 2012; d C-CKD-EPIscr, CKD-EPIscr equation modified for China; e C-CKD-EPIcys, CKD-EPIcys equation modified for China; f C-CKD-EPIscr-cys, CKD-EPIscr-cys equation modified for China. GFR was measured in $\mathrm{mL} / \mathrm{min} / 1.73 \mathrm{~m}{ }^{2}$. 


\section{Kidney \\ Blood Pressure \\ Research}

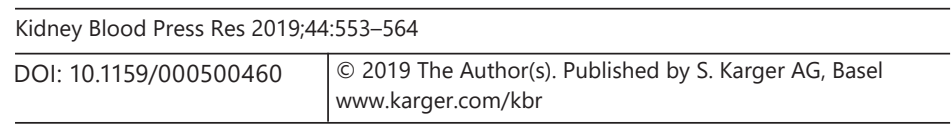

Yang et al.: Revised Equations to Estimate GFR from Scr and Cystatin C in China

accuracy with the CKD-EPI equations. The coefficients to modify CKD-EPIscr, CKD-EPIcys, and CKD-EPIscr-cys for the Chinese population were $0.871,0.879$, and 0.891 , respectively. The coefficient to modify CKD-EPIcys for Chinese female was 0.999 , almost the same as 1.0 , so we omitted the female coefficient. It is suggested that women's coefficient be unnecessary for cystatin C-based GFR estimation. Other studies also showed that cystatin C was independent of sex [13-15].

We observed that C-CKD-EPIcys was the most accurate with the highest P30 value (62.3\%), relative lowest IQR (15.45), and RMSE (0.80) among 6 equations. C-CKD-EPIscr equation was improved in bias $(p<0.001)$, precision, and accuracy ( $p=0.004$ for P30). C-CKDEPIscr-cys equation was also improved in bias $(p<0.001)$, precision, and accuracy $(p<0.001$ for P30). There has been a distinct improvement in the 3 modified equations according to the fit of the data.

There is some literature on cystatin C stating that it is nearly independent of age, sex, and race. Many cystatin $C$ equations that have been proposed in the past only had one variable (cystatin C) in the equation [13-15]. The CAPA equation stands for "Caucasian Asian Paediatric and Adult" cohort and only contains cystatin C and age in the equation [14]. Recently, the new FAS equation (full age spectrum) [16] has shown to be applicable to cystatin $\mathrm{C}$ using a fixed normalization factor for cystatin C $(0.82 \mathrm{mg} / \mathrm{L}$ for subjects up to 70 years and 0.95 $\mathrm{mg} / \mathrm{L}$ beyond that age), independent of sex and probably of race. Further study on the FAS equation for serum creatinine and serum cystatin C implied that the CKD-EPIcysC equation shows serious bias compared to measured GFR [15]. However, evaluation of the CAPA and FAS equations in a diverse population [17] indicates that these newer GFR estimating equations are not more accurate than the CKD-EPI equations in adults. Levey et al. [17] concluded that "current evidence does not support routinely reporting eGFR in adults using these newer equations rather than the CKD-EPI equations." A study recently showed that [18] the performance of the FASscr-cys equation is better than that of the CKD-EPIscr-cys equation in the Chinese population. Whether FASscr-cys equation is better than our modified CKD-EPIscrcys equation or CKD-EPIcys equation needs further research.

Cystatin C is a 13-KD protein that is freely filtered by glomerulus, reabsorbed and catabolized by the renal tubule. It is produced at a constant rate by all nucleated cells and distributed throughout the extracellular fluid. Compared to creatinine, serum cystatin $\mathrm{C}$ is less influenced by muscle mass or diet. Some errors due to the non-GFR determinants of creatinine and cystatin $C$ are smaller in an equation that uses both markers than in an equation that uses only one marker. The KDIGO guideline suggests that cystatin $C$ should be measured in adults with an eGFRscr of $45-59 \mathrm{~mL} / \mathrm{min} / 1.73 \mathrm{~m}^{2}$ who do not have albuminuria [19]. The use of cystatin $\mathrm{C}$ strengthens the association between the eGFR and the risks of death [20]. CKD-EPIcys equation performed well in Japan, which suggested that cystatin C-based equations can be used in patients with different races in whom race modification is not needed [21]. The Japanese coefficient for CKD-EPIscr-cys was 0.908 and showed good performance in Japanese. The coefficient to modify CKD-EPIscr-cys for our Chinese population was 0.891 , which was similar to Japanese coefficient for CKD-EPIscr-cys. Our study indicated that C-CKD-EPIscr-cys equation was improved in bias, precision, and accuracy.

Chronic illnesses such as diabetes mellitus, dyslipidemia, and CKD are relatively silent and rely on the clinical laboratory for diagnosis, particularly in their early stages. Probably, there is no other disease similar to CKD in which patients experience greater loss of organ function before symptoms start to appear [22], so evaluation of GFR for CKD is of paramount importance. The KDIGO guideline suggests using the 2012 CKD-EPI cystatin C and 2012 CKD-EPI creatinine-cystatin C equations, respectively, or alternative cystatin C-based GFR estimating equations if they have been shown to improve accuracy of GFR estimates compared to the 2012 CKD-EPI cystatin C and 2012 CKD-EPI creatinine-cystatin C equations [19]. 


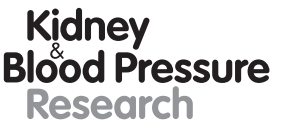

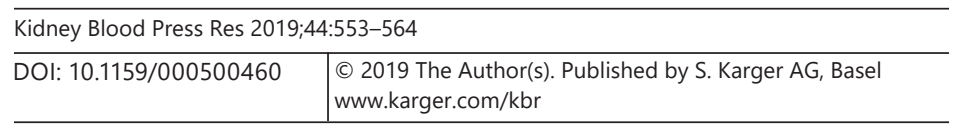

Yang et al.: Revised Equations to Estimate GFR from Scr and Cystatin C in China

C-CKD-EPIcys and C-CKD-EPIscr-cys equations outperformed 2012 CKD-EPI equations for Chinese in our study. It all starts with screening and detection of a silent disease, which give years of opportunity for discovery and modification of its natural history [22].

The CKD-EPI investigators assessed accuracy as P30, which takes into account greater errors at higher values and the absolute values of the difference between measured and eGFR [23]. For this reason, most GFR equation studies take P30 for accuracy. Although other parameters (such as P10 and P20, representing the percentages of estimations falling within \pm 10 and $\pm 20 \%$ of measured GFR, respectively) have also been used, P30 deserves special consideration. It is worth noting that P30 was defined in the absence of any clinical or statistical rationale for considering it an acceptable margin of error [24].

There are also some limitations of this study. First, we use dynamic renal imaging as the rGFR method and may result in great bias. The Nephrology Conference held in 1996 in the USA recommended the adoption of the 2-plasma method [25]. However, the GFR obtained by the modified formula for the calculation of GFR used in this article was derived from studies, calibrated with dual plasma sample ${ }^{99 \mathrm{~m}} \mathrm{Tc}$-DTPA GFR, and reflected kidney function more accurately, and was closer to the true value than those that did not calibrated with dual plasma method [12]. DTPA renal dynamic imaging method was more practical and widely used in routine clinic than dual plasma method [26]. Second, we validated the above 6 equations from 313 participants. The number of validation participants is small. Third, all study participants had CKD. The equations are based on data from Chinese CKD patients; thus, it is not clear whether it is applicable to healthy population. Fourth, extrarenal elimination of serum cystatin $\mathrm{C}$ was not measured.

In summary, our study demonstrated that C-CKD-EPIcys was the most accurate with the highest P30 value, relative lowest IQR, and RMSE among 6 equations. C-CKD-EPIscr and C-CKD-EPIscr-cys equations were improved in bias, precision, and accuracy compared with CKD-EPIscr and CKD-EPIscr-cys. Other external validation of these equations is needed.

\section{Acknowledgments}

This work was supported by grants from Chinese Society of Nephrology (14050430580), High-level Talents of the Fifteenth "Six Talents Peaks" in Jiangsu Province (WSN-113), and National Natural Science Foundation of China (81400714). The funders had no role in study design, data collection and analysis, decision to publish, or preparation of the manuscript.

\section{Ethical Approval}

All procedures performed in studies involving human participants were in accordance with the Institutional Review Boards of Nanjing First Hospital affiliated to Nanjing Medical University at which the studies were conducted (IRB approval number 86-025-52271039-21) and with the 1964 Helsinki declaration and its later amendments or comparable ethical standards.

\section{Disclosure Statement}

The authors have declared that no conflict of interest exists. 


\section{Kidney Blood Pressure Research}

\begin{tabular}{l|l}
\hline Kidney Blood Press Res 2019;44:553-564 \\
\hline DOI: 10.1159/000500460 & $\begin{array}{l}\text { @ 2019 The Author(s). Published by S. Karger AG, Basel } \\
\text { www.karger.com/kbr }\end{array}$ \\
\hline
\end{tabular}

Yang et al.: Revised Equations to Estimate GFR from Scr and Cystatin C in China

\section{Author Contributions}

The research was designed by X.D. All authors helped to write the report and commented on the manuscript. X.D. analyzed the data and advised on statistical issues at the time of the research write up. Y.G. was the research administrator, obtained the data, and prepared communications with participating centers and the data monitoring committee. X.D. and M.Y. were funders. X.D., Y.Z., Y.N., T.L., J.N., and M.Y. were research nurses responsible for recruitment and return of data.

\section{References}

1 Webster AC, Nagler EV, Morton RL, Masson P. Chronic Kidney Disease. Lancet. 2017 Mar;389(10075):123852.

2 Zhang L, Wang F, Wang L, Wang W, Liu B, Liu J, et al. Prevalence of chronic kidney disease in China: a crosssectional survey. Lancet. 2012 Mar;379(9818):815-22.

3 Wang F, Zhang L, Wang H; China National Survey of CKD Working Group. Awareness of CKD in China: a national cross-sectional survey. Am J Kidney Dis. 2014 Jun;63(6):1068-70.

4 Du X, Zhao Y, Huang W, Liu L, Cao C. Association of chronic kidney disease with adverse outcomes. Lancet. $2013 \mathrm{Feb} ; 381(9866): 531-2$.

5 Hallan SI, Matsushita K, Sang Y, Mahmoodi BK, Black C, Ishani A, et al.; Chronic Kidney Disease Prognosis Consortium. Age and association of kidney measures with mortality and end-stage renal disease. JAMA. 2012 Dec;308(22):2349-60.

6 Nitsch D, Grams M, Sang Y, Black C, Cirillo M, Djurdjev O, et al.; Chronic Kidney Disease Prognosis Consortium. Associations of estimated glomerular filtration rate and albuminuria with mortality and renal failure by sex: a meta-analysis. BMJ. 2013 Jan 29;346:f324.

7 Grams ME, Chow EK, Segev DL, Coresh J. Lifetime incidence of CKD stages 3-5 in the United States. Am J Kidney Dis. 2013 Aug;62(2):245-52.

8 Vassalotti JA, Piraino B. Loss of kidney function with aging is a patient safety hazard. Am J Kidney Dis. 2013 Aug;62(2):217-9.

9 Yang M, Xu G, Ling L, Niu J, Lu T, Du X, et al. Performance of the creatinine and cystatin C-based equations for estimation of GFR in Chinese patients with chronic kidney disease. Clin Exp Nephrol. 2017 Apr;21(2):236-46.

10 Inker LA, Schmid CH, Tighiouart H, Eckfeldt JH, Feldman HI, Greene T, et al.; CKD-EPI Investigators. Estimating glomerular filtration rate from serum creatinine and cystatin C. N Engl J Med. 2012 Jul;367(1):20-9.

11 Du X, Liu L, Hu B, Wang F, Wan X, Jiang L, et al. Is the Chronic Kidney Disease Epidemiology Collaboration fourlevel race equation better than the cystatin C equation? Nephrology (Carlton). 2012 May;17(4):407-14.

12 Li Q, Zhang CL, Fu ZL, Wang RF, Ma YC, Zuo L. Development of formulae for accurate measurement of the glomerular filtration rate by renal dynamic imaging. Nucl Med Commun. 2007 May;28(5):407-13.

13 Björk J, Grubb A, Larsson A, Hansson LO, Flodin M, Sterner G, et al. Accuracy of GFR estimating equations combining standardized cystatin C and creatinine assays: a cross-sectional study in Sweden. Clin Chem Lab Med. 2015 Feb;53(3):403-14.

14 Grubb A, Horio M, Hansson LO, Björk J, Nyman U, Flodin M, et al. Generation of a new cystatin C-based estimating equation for glomerular filtration rate by use of 7 assays standardized to the international calibrator. Clin Chem. 2014 Jul;60(7):974-86.

15 Pottel H, Delanaye P, Schaeffner E, Dubourg L, Eriksen BO, Melsom T, et al. Estimating glomerular filtration rate for the full age spectrum from serum creatinine and cystatin C. Nephrol Dial Transplant. 2017 Mar;32(3): 497-507.

16 Pottel H, Hoste L, Dubourg L, Ebert N, Schaeffner E, Eriksen BO, et al. An estimated glomerular filtration rate equation for the full age spectrum. Nephrol Dial Transplant. 2016 May;31(5):798-806.

17 Levey AS, Tighiouart H, Simon AL, Inker LA. Comparing Newer GFR Estimating Equations Using Creatinine and Cystatin C to the CKD-EPI Equations in Adults. Am J Kidney Dis. 2017 Oct;70(4):587-9.

18 Yong Z, Li F, Pei X, Liu X, Song D, Zhang X, et al. A comparison between 2017 FAS and 2012 CKD-EPI equations: a multi-center validation study in Chinese adult population. Int Urol Nephrol. 2019 Jan;51(1):139-46.

19 Kidney Disease: Improving Global Outcomes (KDIGO) CKD Work Group. KDIGO 2012 Clinical Practice Guideline for the Evaluation and Management of Chronic Kidney Disease. Kidney Int. 2013;3(Suppl):1-150.

20 Shlipak MG, Matsushita K, Ärnlöv J, Inker LA, Katz R, Polkinghorne KR, et al.; CKD Prognosis Consortium. Cystatin C versus creatinine in determining risk based on kidney function. N Engl J Med. 2013 Sep;369(10): $932-43$.

21 Horio M, Imai E, Yasuda Y, Watanabe T, Matsuo S; Collaborators Developing the Japanese Equation for Estimated GFR. GFR estimation using standardized serum cystatin C in Japan. Am J Kidney Dis. 2013 Feb;61(2): $197-203$. 
22 Sherwood M, McCullough PA. Chronic kidney disease from screening, detection, and awareness, to prevention. Lancet Glob Health. 2016 May;4(5):e288-9.

23 Levey AS, Stevens LA, Schmid CH, Zhang YL, Castro AF 3rd, Feldman HI, et al.; CKD-EPI (Chronic Kidney Disease Epidemiology Collaboration). A new equation to estimate glomerular filtration rate. Ann Intern Med. 2009 May;150(9):604-12.

24 Porrini E, Ruggenenti P, Luis-Lima S, Carrara F, Jiménez A, de Vries AP, et al. Estimated GFR: time for a critical appraisal. Nat Rev Nephrol. 2019 Mar;15(3):177-90.

25 Blaufox MD, Aurell M, Bubeck B, Fommei E, Piepsz A, Russell C, et al. Report of the Radionuclides in Nephrourology Committee on renal clearance. J Nucl Med. 1996 Nov;37(11):1883-90.

26 Yuan X, Zhang J, Tang K, Quan C, Tian Y, Li H, et al. Determination of Glomerular Filtration Rate with CT Measurement of Renal Clearance of Iodinated Contrast Material versus 99mTc-DTPA Dynamic Imaging "Gates" Method: A Validation Study in Asymmetrical Renal Disease. Radiology. 2017 Feb;282(2):552-60. 